\title{
Kompetenz \\ und Kommunikation
}

\author{
Thomas Dane
}

Wohlfahrtsverbände kennen in der Regel ihre Mitgliedsorganisationen genau. Gerät ein Träger in Not, sind sie deshalb in besonderem Maße zur Krisenhilfe aufgefordert. Dies verlangt hohe fachliche und wirtschaftliche Kompetenz sowie eine vertrauensvolle Kommunikation.

I.

Wenn die Mitgliedsorganisation eines Wohlfahrtsverbandes in eine wirtschaftliche Krise gerät, ist auch der Wohlfahrtsverband gefordert. Die konkreten Unterstützungsmöglichkeiten des Verbandes in der Krisensituation sind jedoch in hohem Maße durch die Wahrnehmung des Verbandes durch das Mitglied und die Wahrnehmung des Mitglieds durch den Verband geprägt. Nachfolgend wird davon ausgegangen, dass der Verband ein reiner Mitgliederverband ist, also nicht als Betreiber von Einrichtungen und Diensten in Konkurrenz zu seinen Mitgliedern tritt.

II.

Die Hauptaufgaben eines Wohlfahrtsverbandes im Verhältnis zu seinen Mitgliedern sind

- die Information der Mitglieder über alle für sie wichtigen Entwicklungen im Hinblick auf die rechtlichen, wirtschaftlichen und politischen Rahmenbedingungen

- die Beratung in fachlichen, organisatorischen, rechtlichen und finanziellen Fragen

- die Förderung des Austausches unter den Mitgliedern, um Problemlösungen nach dem Best- practice-Prinzip zu finden

- die Interessenvertretung der Mitglieder gegenüber öffentlicher Verwaltung und Sozialleistungsträgern insbesondere im Hinblick auf die Gestaltung der Finanzierung sozialer Dienstleistungen

- das politische Lobbying und die Initiierung von Kampagnen zur Beeinflussung der öffentlichen Meinung.

Die zunehmende Dynamik der Veränderung der Rahmenbedingungen für die Erbringung sozialer Dienstleistungen bewirkt, dass die an den Verband herangetragenen Probleme und Fragestellungen an Komplexität gewinnen. Traditionell haben Wohlfahrtsverbände in ihrer Organisation die bürokratisch-funktionale Struktur öf-

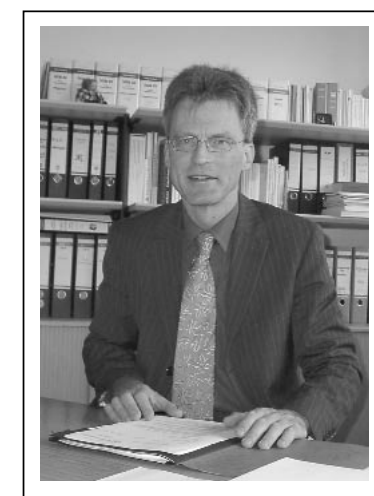

Thomas Dane (45) ist Vorstandsmitglied des Diakonischen Werkes Berlin-Brandenburg-schlesische Oberlausitz, einem Wohlfahrtsverband mit etwa 100 Mitarbeiterinnen und Mitarbeitern. Der Verband vertritt als größter ostdeutscher Landesverband der Freien Wohlfahrtspflege die Interessen von 400 Trägern mit 50.000 Beschäftigten in drei Bundesländern Berlin, Brandenburg und Sachsen. In Hamburg geboren studierte Thomas Dane nach einjährigem Auslandsaufenthalt in Israel Soziologie. Noch vor Ende des Studiums trat er eine Referentenstelle beim Paritätischen Wohlfahrtsverband in Hamburg an, wechselte dann 1995 zur Diakonie nach Berlin-Brandenburg, wo er 1997 zum Vorstandsmitglied berufen wurde. Obwohl Soziologe verantwortet Thomas Dane beim Diakonischen Werk den kaufmännischen Bereich und ist nebenberuflich als Dozent im Fach Betriebswirtschaftslehre tätig. Dies ist darauf zurückzuführen, dass er bereits beim Berufseinstieg die Unterscheidung in Fachleute und Betriebswirte als hinderlich für den Erfolg sozialer Unternehmen empfand. Er hat sich deshalb frühzeitig in der Aushandlung von Leistungsentgelten engagiert und intensiv mit der betriebswirtschaftlichen Steuerung sozialer Unternehmen beschäftigt.

E-Mail Dane.T@diakoniebb.de

fentlicher Verwaltungen gespiegelt. Diese Struktur ist nicht geeignet, die Problemlösungskompetenz von Wohlfahrtsverbänden zu befördern. Vielmehr sind Wohlfahrtsverbände gut beraten sich als moderne Dienstleister zu profilieren, also Anfragen aus dem Mitgliederbereich zunächst anzunehmen und dann unter Bündelung der vorhandenen Ressourcen rasch zu beantworten.

Dies bedeutet, dass für die Mitarbeiterin oder den Mitarbeiter des Verbandes nicht die traditionelle Frage »Bin ich zuständig? « im Mittelpunkt stehen darf. Diese ist Merkmal bürokratischer Verwaltungsstrukturen. Sondern die Kernfrage muss lauten: »Wie beantworte ich die Anfrage 
des Mitgliedes möglichst rasch und welche Kollegin, welchen Kollegen muss ich einbinden, um die Anfrage des Mitgliedes so gut wie möglich zu beantworten?« Die Mitarbeiterin oder der Mitarbeiter übernimmt so in jedem Einzelfall die Verantwortung dafür, dass dem Anliegen des Mitgliedes entsprochen wird: Es wird nicht an die Kollegin oder den Kollegen verwiesen, sondern die erfolgreiche Bearbeitung des Anliegens durch den Verband als Ganzes sichergestellt. Dieses Selbstverständnis ist Voraussetzung dafür, dass ein Verband in der Krise seiner Mitgliedsorganisation als hilfreicher Partner akzeptiert wird.

III.

Ein Verband ist keine Unternehmensberatung. Dies hat folgende Gründe:

- Die Unternehmensberatung lässt sich ihre Arbeit nach Aufwand bezahlen. Erwartet der Kunde hohen Aufwand, muss er viel zahlen. An den Verband leistet das Mitglied einen pauschalen Mitgliedsbeitrag, der unabhängig vom konkreten Aufwand im Einzelfall ist. Eine Intensivberatung des einzelnen Mitgliedes kann der Verband deshalb nicht leisten.

- Die Unternehmensberatung wird für einen konkreten kurzfristigen Zeitraum in Anspruch genommen. Verband und Mitglied stehen dagegen in einem langfristigen Kommunikationsprozess. Während die Unternehmensberatung die Situation systematisch analysiert und dann einen konkreten Lösungsvorschlag entwickelt, analysiert der Verband laufend - wenn auch nicht immer systematisch - und macht ebenso laufend Vorschläge. Deshalb ist ein Verband in der Krise eines Mitgliedes nie neutral, weil er die Entstehung der Krise bereits verfolgt hat und Ursachen bereits im Vorfeld bewertet hat.

- Die Unternehmensberatung hat die Chance in der Krisensituation einen Lösungsvorschlag zu machen. Wenn dieser erfolgreich umgesetzt wird, wird der Erfolg der Arbeit der Unternehmensberatung zugerechnet. Der Verband dagegen wird für die Entstehung der Krise in die Mitverantwortung genommen, beispielsweise weil er sie nicht verhindert hat oder es ihm nicht gelungen ist, bessere Rahmenbedingungen zu schaffen. Er kann durch eine erfolgreiche Beratung sein »Sündenregister « lediglich abbauen.

Ein Verband ist deshalb in seiner Arbeit nicht auf Krisenbewältigung, sondern auf prophylaktische Krisenvermeidung ausgerichtet: Er versucht künftige Entwicklungen prognostisch zu beschreiben und strategische Ausrichtungen zu empfehlen. Er berät teilweise ohne Auftrag und weiß sich mitverantwortlich für die erfolgreiche Entwicklung seiner Mitglieder.

\section{IV.}

Träger sozialer Einrichtungen und Dienste in der Krise haben in der Regel entweder keine Erwartungen an den Verband oder ein angespanntes Verhältnis zu ihm. Im er- sten Fall wird entweder die primäre Ansprechperson im Verband nicht als kompetent angesehen, genießt also generell keine Akzeptanz im Mitgliederbereich. Dann besteht Handlungsdruck beim Verband, sich von dieser Ansprechperson so schnell wie möglich zu trennen, weil deren fehlende Akzeptanz unmittelbar dem Verband zugeschrieben wird. Oder aber es besteht keine hinreichende Nähe zwischen Mitglied und Verband. Dann muss der Verband prüfen, ob seine Organisation sachgerecht ist oder ob nicht Defizite in der Verbandsorganisation zu dieser fehlende Nähe geführt haben. Im zweiten Fall, dem angespannten Verhältnis zum Verband, ist die Ursache in der Regel, dass bereits in der Entstehung der Krise aus dem Verband heraus die Entwicklung des Mitgliedes kritisch hinterfragt worden ist.

Ein Verband ist auch eine Kommunikationsplattform. Stellt ein Mitglied fest, dass es durch den Verband kritisch wahrgenommen wird, dann entsteht rasch die Befürchtung, der Verband trage diese Einschätzung weiter. Dadurch würden der Status des Mitgliedes und das Ansehen der dieses Mitglied vertretenden Personen sinken. Dies führt dazu, dass sich das Mitglied vom Verband distanziert und die Kommunikation mit dem Verband einschränkt.

Deshalb darf der Verband einer kritischen Wahrnehmung des Mitglieds nicht offensiv Ausdruck verleihen, da dann die Kommunikationsstränge gekappt werden. Hält er sich jedoch mit kritischen Äußerungen zurück, wird er in die Mithaftung für die Entwicklung des Mitgliedes ge-

\section{"Liquidität, Bilanzsituation, Prozessqualität«}

»Krisenintervention, wie wir sie betreiben - nämlich an der Basis - ist sehr simpel. Drei Faktoren prüfen wir in jeder Situation sofort: die Liquidität, die Bilanzsituation, die Prozessqualität. Im ersten Sanierungsschritt stellen wir die Handlungsfähigkeit sicher, insbesondere die nach außen, das bedeutet, wir >liefern< im Wesentlichen Liquidität. Wir stützen mit Liquidität und überzeugen dadurch die Geldgeber. Dies könnten wir nicht, wenn wir nicht operative Erfahrung hätten. Wir haben Mitarbeiter, die das operative Tagesgeschäft kennen. Unsere erste Analyse dauert nicht länger als zwei Tage. Wir gehen gleichzeitig vor: Die Analyse von existenziell wichtigen Stellen und die Behebung der großen Störfaktoren an diesen Stellen erfolgen nahezu simultan. Ein Beispiel ist die Pflegequalität: Nicht die aktuelle Belegung eines Pflegeheims bringen die Lawine ins Rutschen, sondern echte Gesundheitsrisiken, die schließlich auch über eine Prüfung des MDK zur Schließung der Einrichtung führen können. Hier muss man rechtzeitig eingreifen und die Lawine stoppen, und zwar durch Gespräche mit relevanten Partnern wie dem MDK und der Heimaufsicht.«

Bernd Anders, Landesgeschäftsführer des Deutschen Roten Kreuzes in Niedersachen. In: "Krisenintervention heißt begleiten bis zum Erfolg! « Gespräch mit Bernd Anders, Landesgeschäftsführer des Deutschen Roten Kreuzes in Niedersachsen. SOZIALwirtschaft 3/2007, Seite 13-15. 
nommen. Diese Gratwanderung zwischen Kritik und Mithaftung kann nur durch besonders intensive Kommunikation bewältigt werden. Denn mit steigender Intensität der Kommunikation eröffnen sich zunehmend Möglichkeiten, Kritik in Form des wohlmeinenden Rates konstruktiv zum Ausdruck zu bringen. Hieraus ergibt sich ein sehr komplexes Anforderungsprofil an die Mitarbeiterinnen und Mitarbeiter des Verbandes.
- fehlende Marktnähe und unzureichende Anpassung der Leistungen an die Bedürfnisse der Kunden

- unzureichende fachliche Kompetenz oder Zusammenarbeit der Leitungspersonen

- fehlendes stabiles Kerngeschäft des Trägers oder zu breit gefächertes Leistungsprofil (»Gemischtwarenladen«)

\section{"Ein Wolfahrtsverband ist nicht auf Krisenbewältigung, sondern auf Krisenvermeidung ausgerichtet «}

- langfristige Quersubventionierung unprofitabler Einrichtungen

- aufgeblähter aus Umlagen finanzierter Verwaltungskopf

- zentralistische Strukturen und Leitungskräfte ohne Kompetenz und Verantwortung auf der dezentralen Ebene
Bei Meinungsunterschieden zwischen Mitglied und Verband bieten sich dem Mitglied auch auf der emotionalen Ebene einige Argumente, die helfen, den Verband auf Distanz zu halten. Dies kann zum Beispiel der Vorwurf sein, der Verband wolle nur Einfluss auf das Mitglied gewinnen und Macht ausüben oder gar zum eigenen Vorteil in die Geschäfte des Mitglieds hineinreden. Derartige sachlich nicht begründete Abwehrhaltungen dienen dazu, eine Solidarisierung der Akteure beim Mitglied gegen eine Einflussnahme des Verbandes zu bewirken. Der Verband kann dem jedoch entgegenwirken: Dies geschieht dadurch, dass die Persönlichkeit und Fachkompetenz des verbandlichen Vertreters keine Angriffsfläche bieten.

Schließlich muss sich der Verband häufig organisatorische Defizite des Mitgliedes zurechnen lassen. Wenn etwa der Informationsfluss zwischen dem Mitglied und seinen dezentralen Einrichtungen nicht funktioniert, entsteht auf Einrichtungsebene häufig der Eindruck, dass die unzureichende Information des Verbandes das eigentliche Problem ist. Mancher Träger scheut sich nicht, wider besseres Wissen diesen Eindruck zu verstärken, um selbst aus der Schusslinie zu kommen.

v.

Die Mitarbeiterinnen und Mitarbeiter des Verbandes kennen in der Regel die Mitglieder sehr genau. Bei hinreichender eigener Qualifikation sind sie in der Lage, für jedes Mitglied auf Anhieb ein Stärken-Schwächen-Profil zu erstellen sowie Chancen und Risiken der künftigen Entwicklung des Mitgliedes zu beschreiben. Krisen von Mitgliedern sehen sie deshalb langfristig kommen und versuchen bereits weit vor dem Eintritt der Krise dieser entgegenzuwirken.

Die Ursachen von Krisen sind häufig vergleichbar. Einige typische Ursachen seien hier kurz angeführt:

- Einmischung von ehrenamtlicher Leitung in das Tagesgeschäft bei zugleich unzureichender Kompetenz

- fehlende Kompetenz der Aufsichtsorgane in der Kontrolle der hauptamtlichen Leitung

- fehlende strategische Ausrichtung
- Überforderungen und Engpasssituationen in Leitungsfunktionen aufgrund von Organisationsdefiziten

- Unterkapitalisierung oder zu geringe Größe um Schwankungen im Umsatz zu kompensieren

- falsche Finanzierung von Investitionsmaßnahmen oder

- Fehler in der Ausgestaltung langfristiger Verträge.

Diese Defizite können in der Regel weit vor Eintritt der Krise festgestellt werden. Der Verband kann hier versuchen, durch wohlmeinenden Rat bei der rechtzeitigen Beseitigung möglicher Ursachen späterer Krisen zu helfen.

Der Verband trifft jedoch vor Eintritt der Krise häufig auf Beratungsresistenz bei der Mitgliedsorganisation. In dieser Situation ist es wichtig, dass der Verband das Vertrauen des Trägers nicht durch zu heftiges Einfordern von Maßnahmen belastet, denn dann wird er bei einer Zuspitzung der Krise nicht mehr hinzugezogen: Die Verantwortlichen beim Träger befürchten dann, der Verband mache deutlich, dass sie für die Krise verantwortlich sind, weil sie vorab auf Fehler hingewiesen worden sind, diese aber trotzdem nicht abgestellt haben.

Der Verband ist in einer solchen Situation gut beraten, seine Kritik in positive Vorschläge zu fassen. Er kann seine Feststellungen andererseits nämlich nicht verschweigen: Dann würde bei Eintritt der Krise auch seine Kompetenz in Frage gestellt.

Die Beratungsresistenz des Mitgliedes ist häufig darauf zurückzuführen, dass Meinungsbildungsprozesse bei Trägern komplex sind. Über notwendige Maßnahmen muss jedoch Einvernehmen hergestellt werden. Wenn jedoch in den Entscheidungsorganen des Trägers $\mathrm{zu}$ viele Personen vertreten sind, die gar nicht in der Lage sind, etwa die wirtschaftlichen Zusammenhänge zu begreifen, dann wird Meinungsbildung in den Organen des Trägers zum Lotteriespiel. Hier kann es zweckmäßig sein, dass der Verband in die direkte Kommunikation mit den Organen des Trägers eintritt und unter Einsatz seiner Fachkompetenz bei der Meinungsbildung behilflich ist. Dies setzt jedoch voraus, dass er aus den Organen heraus um diese Unterstützung gebeten wird. 
VI.

Ressourcen abzuhelfen. Er kann allenfalls den einen oder anderen Zuschuss vermitteln. Seine Kernleistung ist jedoch die Beratung beim Abstellen der Krisenursachen.

Wie oben ausgeführt kann nach Eintritt der Krise die Hauptaufgabe des Verbandes nicht in der intensiven Krisenbewältigung oder dem Interimsmanagement liegen. Dies überfordert zwar nicht unbedingt die Kompetenzen, aber stets die Ressourcen des Verbandes. Außerdem sehen es andere Mitgliedsorganisationen regelmäßig kritisch, wenn sich der Verband verstärkt bei einem Mitglied engagiert: Denn auch unter den Mitgliedern eines Verbandes gibt es Wettbewerb.

Die wichtigste Funktion des Verbandes liegt in der Unterstützung bei der Durchsetzung notwendiger strategischer Entscheidungen für die Fortführung der Geschäfte des Trägers: also in der Beratung und Bestärkung der Organe. Die Organe des Trägers sind in der Krise in der Regel hilflos: Denn sonst wäre diese Krise nicht eingetreten. Außerdem stehen die Organe in der Kritik, weil sie für die Krise verantwortlich gemacht werden. In dieser Situation kann der Verband die Organmitglieder schützen und stützen, indem er sich auf deren Seite stellt. Es ist eine Art Tauschgeschäft: Der Verband stützt die Trägerorgane und im Gegenzug billigen die Trägerorgane dem Verband eine aktive Rolle bei der Vorbereitung notwendiger Organentscheidungen zu.

Die Vorgehensweise des Verbandes entscheidet sich in der Krisensituation von der eines externen Beraters. Der Verband versucht, so schnell wie möglich alle ihm verfügbaren Informationen zusammenzutragen und ergänzende Informationen beim Träger abzufordern. Dann erstellt er aus diesen Informationen, seinen Erfahrungen in vergleichbaren Situationen und der meist hervorragenden Kenntnis der Rahmenbedingungen eine Art Sanierungsskizze oder entwickelt ein Notprogramm. Dieses wird dann den Trägerorganen präsentiert. Zugleich wird eine Empfehlung ausgesprochen, an welchen Stellen die Inanspruchnahme externer Berater erfolgen sollte. Anschließend wird aktiv Überzeugungsarbeit in den Trägerorganen geleistet.

Der Vorteil für das Mitglied liegt darin, dass der Verband mitten in der Krise sofort da ist, sehr rasch und ohne zusätzliche Kosten die Handlungsfähigkeit der Trägerorgane wieder herzustellen hilft. Und er legt ein Handlungskonzept, einen Verfahrensvorschlag vor. Dabei ist es in dieser Situation nicht entscheidend, ob jede einzelne empfohlene Maßnahme »richtig « ist. Wichtig ist, dass die Richtung stimmt und die Machbarkeit der Krisenbewältigung realistisch eingeschätzt wird. Die dafür benötigte Kompetenz muss dem Verband zugestanden werden, dieses Vertrauen gegeben sein. Aufseiten des Verbandes kann es hier sinnvoll sein, den regulären langjährigen Ansprechpartner des Trägers beim Verband aus der vordersten Linie zurückzuziehen und über eine andere Person die Krisenbewältigung zu organisieren. Dies ermöglicht einen deutlichen Schnitt zur Vorkrisensituation und gegebenenfalls kann der Verband den Wechsel nutzen, um mehr Autorität einzubringen. Verbandsintern kann die Entscheidungsvorbereitung nach wie vor durch die bisherige Ansprechperson wesentlich mit geleistet werden.

Der Verband ist, von Ausnahmen abgesehen, nicht in der Lage, einer Krise durch Einbringen von finanziellen
Natürlich steht er auch während des Prozesses der Krisenbewältigung stets mit Rat und Tat zur Seite. Er ist ständiger Gesprächspartner der Akteure innerhalb der Mitgliedsorganisation, stützt diese und versucht, deren Kompetenz

in der Krisenbewältigung zu entwickeln. 